\title{
Endoscopic tympanoplasty using Tutoplast versus conventional microscopic tympanoplasty
}

\author{
Taha M. Abdelaal ${ }^{*}$ and Ahmed A. Ibrahim
}

\begin{abstract}
Background: Temporalis fascia and perichondrium are still the most successful graft materials for primary microscopic repair of tympanic membrane perforations worldwide with $80 \%$ to $90 \%$ success rate. However, minimally invasive surgery is becoming standard in many fields and the endoscope has been largely incorporated in ear surgery over the past 10 years. This study aimed to evaluate the success rate of endoscopic transcanal

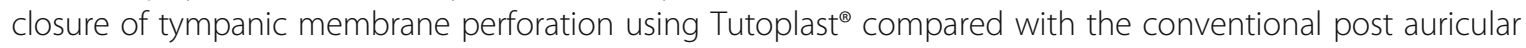
approach using homologous temporalis fascia graft.

Results: Our study was conducted on 50 patients, were equally divided into 2 groups. Group A patients have been operated endoscopic transcanal using the Tutoplast as a graft material. Group B patients have been operated using a temporalis fascia graft through a post-auricular approach. The graft take rate, the pre- and post-operative pure tone audiogram, and operative time were compared in both the groups. The graft uptake rate was 100\% in both groups. The mean of ABG showed significant improvement in both groups. In group A, the mean of ABG was 28.2 pre-operatively and became 12.12 post-operatively. In group B, the mean of ABG was 29.72 pre-operatively then became 15.2 post-operatively. Comparing the mean of post-operative ABG between both groups showed statistically significant difference as $p$ value was 0.000496 . We found a significant difference of the mean average operative time between both groups as the mean average time for group A was 48.08 min compared to 69.4 min for group B with $p$ value 0.049772295 .
\end{abstract}

Conclusions: Endoscopic closure of tympanic membrane perforation using Tutoplast is successful short-time procedure avoiding the postauricular or endaural incisions with shorter operative time.

Keywords: Tutoplast, Endoscopic tympanoplasty, Transcanal approach

\section{Background}

Myringoplasty is one of the commonest otological procedures done by an otolaryngologist to control chronic mucosal otitis media [1]. Berthold was the first one who introduces the thick skin as a graft in 1878. Later on, a graft of mesodermal origin, such as perichondrium, fascia, vein, or fat tissue, was more convenient by otologist in myringoplasty [2]. The outcome of surgical approach may be affected by the perforation size, site, type

\footnotetext{
* Correspondence: tahaabdelaal2020@gmail.com

Department of Otorhinolaryngology, Benha University, Benha, Egypt
}

of the used graft surgeon's experience, and the surgical approach as well [3-5]. Microscopic tympanoplasty was the standard procedure for closure of tympanic membrane perforation since 1950 [6].

Endoscopic tympanoplasty was used since 1990 and because of its wider view, avoiding postsurgical incision with better access through a narrow external canal, was broadly used nowadays. Several studies have been published discussing the endoscopic repair of tympanic membrane perforation by different graft materials [7-9].

Tutoplast ${ }^{\circ}$ fascia temporalis is acellular dehydrated human fascia temporalis graft preserved and sterilized by

\section{Springer Open}

(c) The Author(s). 2021 Open Access This article is licensed under a Creative Commons Attribution 4.0 International License, which permits use, sharing, adaptation, distribution and reproduction in any medium or format, as long as you give appropriate credit to the original author(s) and the source, provide a link to the Creative Commons licence, and indicate if changes were made. The images or other third party material in this article are included in the article's Creative Commons licence, unless indicated otherwise in a credit line to the material. If material is not included in the article's Creative Commons licence and your intended use is not permitted by statutory regulation or exceeds the permitted use, you will need to obtain permission directly from the copyright holder. To view a copy of this licence, visit http://creativecommons.org/licenses/by/4.0/. 
gamma irradiation. It is produced in 4 sizes: $20 \mathrm{~mm}$ spot, $20 \mathrm{~mm} \times 30 \mathrm{~mm}$ strip, $10 \mathrm{~mm}$ spot and $10 \mathrm{~mm} \times$ $25 \mathrm{~mm}$ rounded on one of the strip ends which was used in this study.

Tutoplast $^{\oplus}$ fascia temporalis is acellular dehydrated human fascia and its tissue is selected according stringent specifications with respect to donor selection and serological studies in order to minimize the risk of transmitting infectious diseases and to guarantee optimal biomedical properties. The development of Tutoplast ${ }^{\circ}$ tissue is done using a defined procedure involving the careful cleansing of the tissue and tissue sparing solvent dehydration. This process is subject to a continual quality control. It acts as a guide for the production of vital, vascularized tissue which helps closure of the tympanic membrane.

Our study aimed to evaluate the rate of graft intake, outcome of hearing for endoscopic transcanal closure of tympanic membrane perforation using Tutoplast ${ }^{\oplus}$ com- $^{-}$ pared with the traditional post auricular approach using homologous temporalis fascia graft.

\section{Methods}

This study was conducted on 50 patients after being approved by the medical research ethics committee. Written consent from all patients to be enrolled in this study was obtained and all data were kept confidential.

All selected patients were subjected to preoperative full ORL examination included oto-endoscopic assessment, audiological evaluation in the form of pure tone audiometry (PTA), and air bone gab (ABG) was calculated. CT scan was done for all patients to ensure there is no ossicular disease or any hidden pathology.

Inclusion criteria: (1) Chronic suppurative otitis media with dry central perforation of variable size and shape, with no history of ear discharge for the last 3 months preoperatively. Patient's age ranged from 20 to 48 years with no other comorbidity.

Exclusion criteria: (1) Patients with ossicular disease. (2) Patients with attico-antral disease (attic or marginal perforations, patients with any evidence of cholesteatoma). (3) Patients with revision surgery. (5) Patients with sinonasal pathology. (6) Patients with other comorbidities that may affect the study outcome, e.g., uncontrolled diabetics, renal failure on dialysis.

Patients had been randomly divided into 2 groups $\mathrm{A}$ and $\mathrm{B}$, each included 25. Patients with even birthdate number were assigned under group $\mathrm{A}$, while patients with odd birthdate number were assigned under group B. All cases of both groups were operated by a single surgeon (the second author) to avoid any bias.

Group A included 25 patients whom have been operated endoscopically using the Tutoplast as a graft material and through transcanal approach. Group B included
25 patients whom have been operated using a temporalis fascia graft through the traditional post-auricular approach. Underlay technique was performed for all patients in both groups.

\section{Operative details in group A}

In this study group, we used Tutoplast $10 \mathrm{~mm} \times 25 \mathrm{~mm}$, rounded on one of the strip ends (Fig. 1). It is manufactured by TUTOGEN MEDICAL GmBH IndusterestraBe 6, D-91077 Neunkirchen a. Br. Germany.

Preparation of the graft done during induction of general anesthesia: Tutoplast was prepared by reshaping the graft using a scissor into a suitable-sized piece usually double the size of the perforation, and as recommended by the manufacturer that Tutoplast was rehydrated in sterile, endotoxin-free $0.9 \%$ saline solution for at least 30 min under sterile conditions before use.

Proper positioning of the patient's head as the head was turned to the non-operated side, elevated 30 degrees from the table and the operated ear being upper most and dimming the operating room light as well as adjusting the endoscope light on average power were advised to be followed for all patients before starting the procedure. We used 0 degree, $11 \mathrm{~cm}$ length, $2.7 \mathrm{~mm}$ in diameter rigid endoscope which facilitated easy handling and better control.

Using an ear pick, we started the procedure by refreshing the edge of the perforation all around. Minimal bleeding in this step is controlled by placing a cotton ball soaked in adrenaline 1:200000. After infiltration of the 4 quadrants of external canal with $3 \mathrm{ml}$ of 1:200000 adrenaline solutions. A transcanal incision was created using the rounded curved knife just medial to the bony cartilaginous junction from 1-6 o'clock in the right ear, anticlockwise and from 11-6 o'clock in the left ear, clockwise.

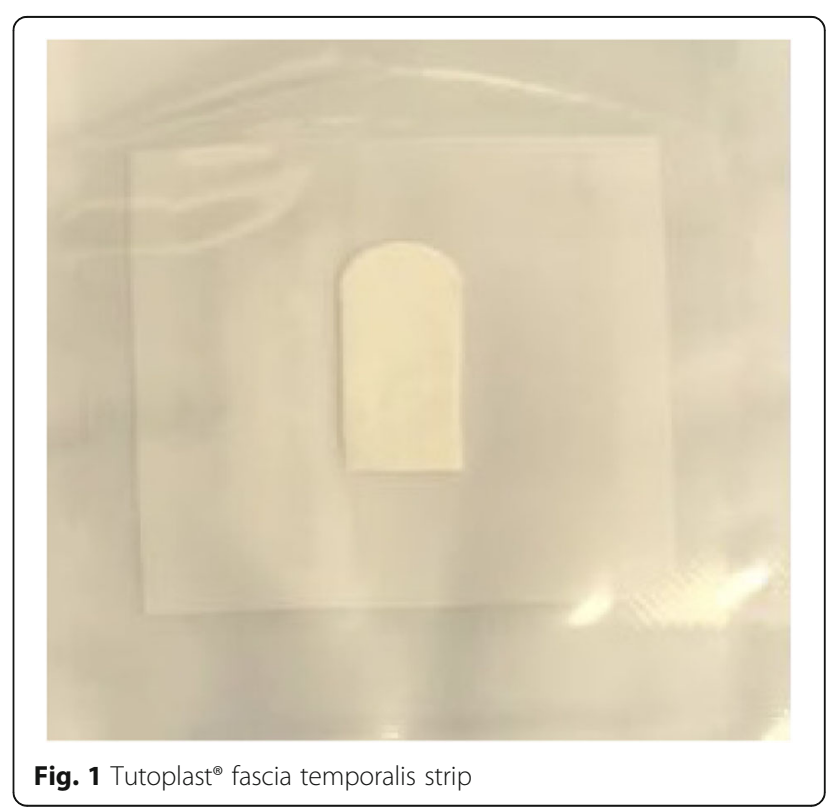


Elevation of skin flap was made using a fine dissector down to the annulus then a round knife was used for little release of the meatal flap superiorly and inferiorly, this helped to reflect the tympanomeatal flap anteriorly. An ear pick was used to separate the annulus from the middle ear mucosa and the drum from the handle of malleus. The handle of malleus was cleaned of any attached epithelial tissues, using micro-crocodile forceps and an ear pick, which allowed good exposure of all middle ear details (Fig. 2). A cotton ball and fine suction were used to control any bleeding, which might affect endoscopic view.

The graft material was introduced under vision of endoscope to underlay the annulus and overlay the handle of malleus (Fig. 3) and then the graft was reflected anteriorly with the tympanomeatal flap. The middle ear was packed carefully using gel foam (Fig. 4). The graft was carefully positioned under the edge of the perforation all around then the posterior tail of the graft was settled over the posterior bony meatal wall and then the tympanomeatal flap was returned over the posterior meatal wall. The endoscope was used to ensure the graft was fitted perfectly under the anterior edge of the perforation (Fig. 5). The external auditory canal was packed with gel foam till the incision site and an ear wick soaked with antibiotic cream was placed.

Group B patients were operated using the surgical microscope and through the conventional post-auricular approach, the graft material used was the patient's temporalis fascia following the basic classic steps of tympanoplasty.

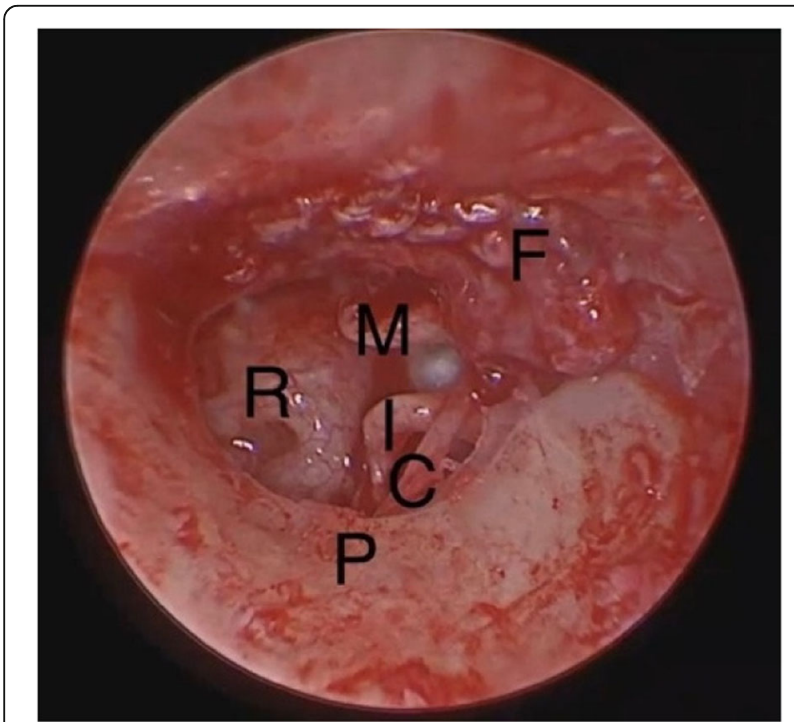

Fig. 2 Endoscopic view of middle ear structures. P, posterior meatal wall; C, chorda tympani; I, incus; $R$, round window; $M$, malleus; $F$, tympanomeatal flap

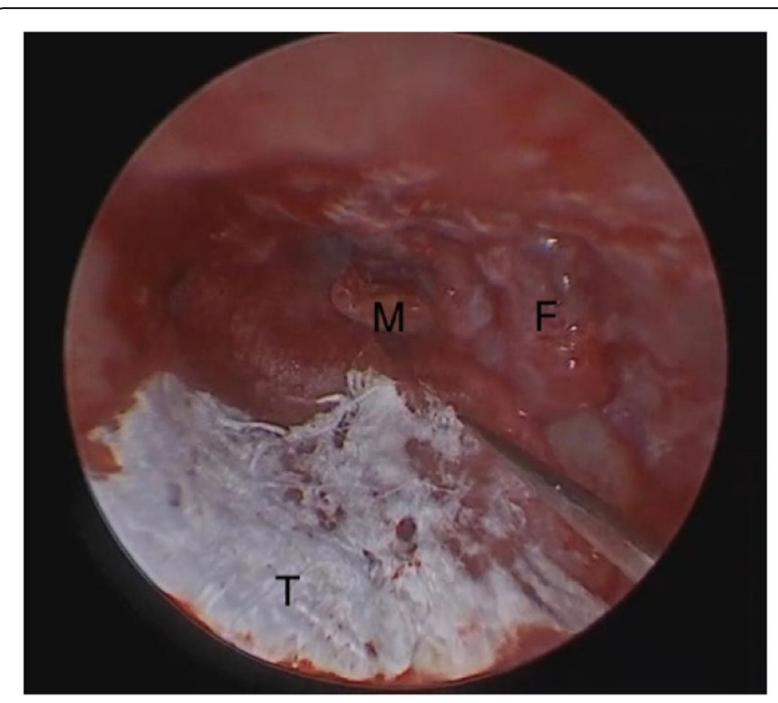

Fig. 3 Underlay of the Tutoplast graft. F, tympanomeatal flap; M, malleus; T, Tutoplast

The surgery duration was calculated by the circulating nurse and recorded for each patient. This duration started from surgical skin incision time till surgery completion time (skin to skin time).

\section{Postoperative follow-up}

All patients were discharged the same day postoperatively without any complication. Postoperative instructions were explained for the patients to avoid any water entrance and care against upper respiratory tract infection. The ear dressing and wick were removed on the first follow up visit one week postoperatively.

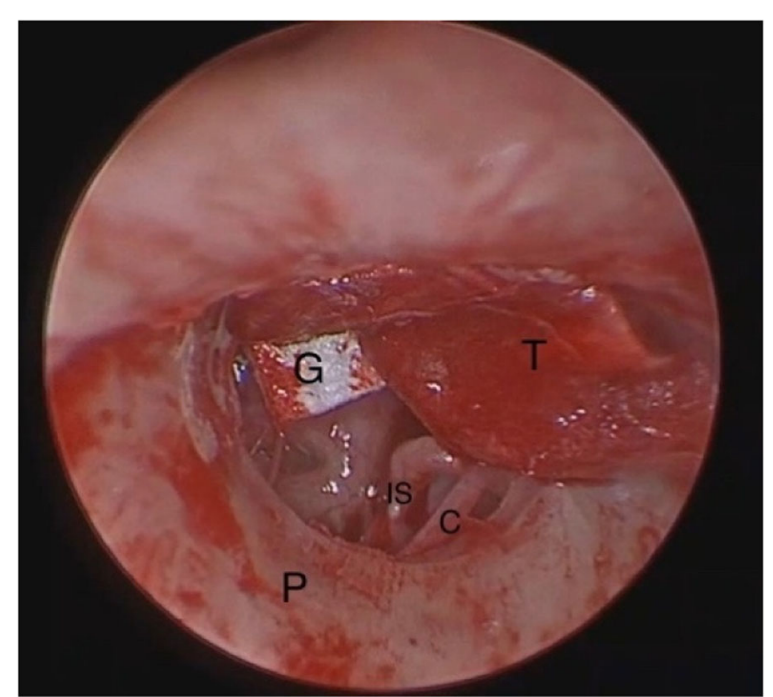

Fig. 4 Packing the middle ear cavity with gelfoam. P, posterior meatal wall; C, chorda tympani; IS, incudo-stapedial joint; M, malleus; T, Tutoplast; G, gelfoam 


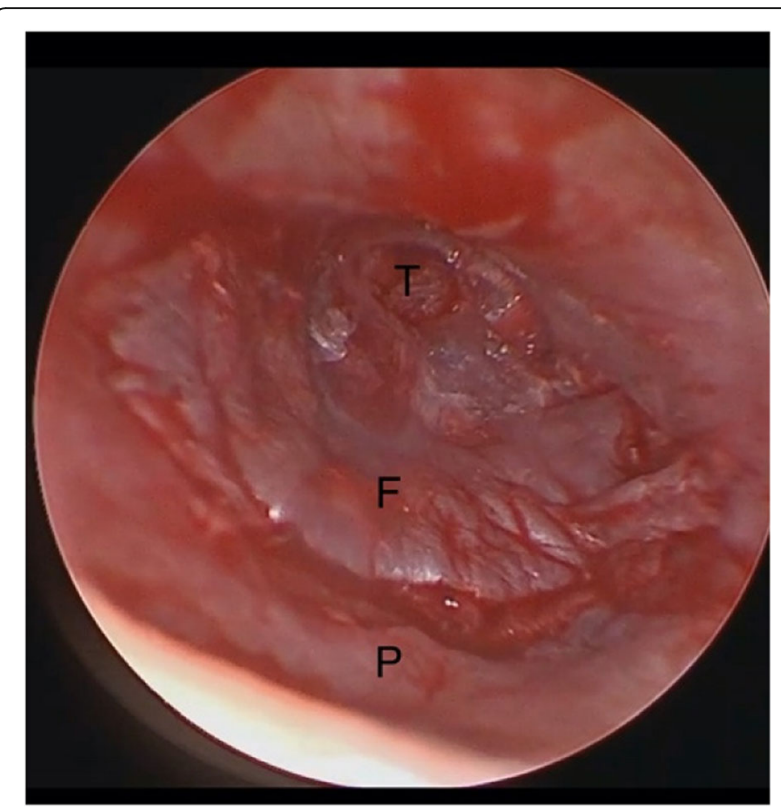

Fig. 5 Final placement of the graft under all edges of the perforation and tympanomeatal flap back. P, posterior meatal wall; F, tympanomeatal flap; T, Tutoplast

Furthermore, all patients were followed up regularly every 3 months for 1 year, in each visit, full ORL examination was performed. One year postoperatively, all cases were examined using oto-endoscope for assessment of the final graft take and PTA was done for each patient and the ABG was calculated and compared with the preoperative one.

After concealing the studied groups, data were sent to the first author for blind outcome assessment and statistical analysis using SPSS version 22 where mean and standard deviation were calculated and unpaired $t$ test was used to compare between two mean to calculate $P$ value.

\section{Results}

This study was conducted on 50 patients with dry central perforations of variable sizes. They included 28 males and 22 females. Their ages ranged from 20 to 48 years with mean age 32 years for group A and 30.8 years for the group B with no significant difference between both groups. They were enrolled in a prospective randomized double-blinded study during the period from January 2017 to January 2020.
For patients of group A, we found that the perforation was repaired successfully through transcanal endoscopic tympanoplasty using Tutoplast as a graft considering tympanic membrane perforation closure with a $100 \%$ take rate with no residual perforation or any faulty healing. PTA results 1 year postoperatively were compared with the preoperative and showed a significant improvement, as the mean of pre-operative ABG was 28.2 and then became 12.12 post-operatively. The surgery duration ranged from 45 to $60 \mathrm{~min}$ with average $48.08 \mathrm{~min}$.

For patients of group B, we found the perforation was repaired successfully using patient's temporalis fascia graft through post-auricular approach with take rate $100 \%$ with no residual perforation. Significant improvement was observed in the pure tone audiometry results post-operatively in comparison with the pre-operative results as the mean pre-operative ABG was 29.72 then became 15.2 post-operatively. The surgery duration ranged from 50 to $90 \mathrm{~min}$ with average $69.4 \mathrm{~min}$.

Unpaired $t$ test was used to calculate the $P$ value. Comparing the mean of pre-operative ABG between both groups, showed insignificant difference as $p$ value was 0.072107 , while comparing the mean of postoperative ABG between both groups showed statistical significant difference as $p$ value was 0.000496 . There was statistically significant difference of the mean average operative time between both groups as the mean average time for group A was 48.08 min compared to $69.4 \mathrm{~min}$ for group B as $p$ value was 0.049772295 (Table 1).

\section{Discussion}

Repair of the tympanic membrane perforation in chronic tubo-tympanic disease is one of the commonest ear surgeries, which is performed in different techniques with different graft materials [10]. The most recent technique is the endoscopic tympanoplasty for repair of tympanic membrane perforation. At first, endoscopes were used as a diagnostic as well as a teaching tool of tympanic membrane and ear canal [11].

Abdulvahap Akyigit et al. summarized the advantages of endoscopic ear surgery to be woundless procedure, with no skin incision, that does not require curettage or canalplasty of external ear canal, provides less postoperative pain, sooner recovery, and provide better cosmetic outcomes [12].

In contrast to our results, Mohindra $\mathrm{S}$ et al. has reported some drawbacks of endoscopic middle ear surgery. $\mathrm{He}$

Table 1 Statistical comparison between both groups as regards the operative time and the pre and post-operative ABG

\begin{tabular}{lllll}
\hline & Group A & Group B & $\boldsymbol{P}$ value & Significant $\boldsymbol{P}<\mathbf{0 . 0 5}$ \\
\hline Operative time & $48.08 \pm 4.47$ & $69.4 \pm 9.919$ & 0.049772295 & Yes \\
Pre-op. ABG & $28.2 \pm 3.617$ & $29.72 \pm 3.623$ & 0.072107 & No \\
Post-op. ABG & $12.12 \pm 2.833$ & $15.2 \pm 3.354$ & 0.00129694 & Yes \\
\hline
\end{tabular}


believed that the endoscopic technique is based on $2 \mathrm{di}-$ mension images, which make the perception difficult; thus, the surgeon has to make sure whether the graft has been sufficiently lifted to make contact with the edge of the perforation. Also, he stated that one-handed technique is one of the shortcomings of endoscopic surgery, as the surgeon has to use one hand to hold the endoscope and the other hand to perform the actual surgery [13]. Similar results by Kozin ED et al. who concluded that endoscopic ear surgery has several disadvantages as compared with microscopic technique. The endoscope may result in direct trauma to the external canal with subsequent bleeding, and the heat produced by endoscopic light might lead to thermal trauma to the middle ear structures and even the inner ear $[14,15]$.

However, we believed that although endoscopic tympanoplasty is a single-handed technique, a welltrained surgeon could efficiently perform it without any trauma using the endoscope with average light intensity and dimming operating room light. In agreement with our beliefs and results, Nayeon Choi1 et al, who indicated in spite of the one-handed endoscopic surgery, it can be successfully performed by an experienced surgeon. Even in patients with large tympanic membrane perforation or anterior bony overhang. Their results showed endoscopic tympanoplasty group had significantly shorter operation time than the microscopic tympanoplasty group. Closure of the postauricular incision in the microscopic postauricular tympanoplasty group might extend the operation time. In addition, the endoscopic technique offered a wide and clean surgical view with minimal canal incision $[15,16]$.

The aim of this study was to evaluate the success rate of Tutoplast graft through transcanal endoscopic approach and to compare it with the results of classic otomicroscopic post auricular tympanoplasty using patient's temporalis fascia graft. In our study, we have believed that endoscopic transcanal tympanoplasty using Tutoplast graft carries a great successful rate with no external wound as well as many advantages for repair of tympanic membrane perforation. It gives surgeon a better view of middle ear details comparing with oto- microscope particularly with advanced technology cameras. Tutoplast like other homograft have the risk of iatrogenic transmission of infectious disease; however, in this study, neither group A nor B patients developed any kind of infectious disease. The only concern of using Tutoplast was its high cost.

In agreement with our study, Sandeep Kumar et al. concluded that endoscopic success myringoplasty has a little steep in comparison with microscopic myringoplasty, even though the leaning curve they recommended is worthwhile due to its clear benefits [17].
The graft success rate in myringoplasty was reported as $80 \%$ to $90 \%$ in literature [18], while in our study, the graft success rate obtained by endoscopic tympanoplasty using Tutoplast was $100 \%$ in the 25 cases done by this technique.

Similar results were obtained in a study conducted by Khaled M. Mokbel et al. in 2015. They performed an endoscopic transcanal cartilage myringoplasty for repair of subtotal tympanic membrane perforation group with $100 \%$ take rate, while the take rate was $90 \%$ for the comparative group performed by traditional microscopic technique. Their mean operative time in the endoscopic group was $40 \pm 5.5 \mathrm{~min}$ compared to $48.08 \mathrm{~min}$ in our study. This difference in the average time between both studies is more likely to be related to training on the endoscopic use [19].

Awad and Hamid reported that operation time is shorter in patients undergoing endoscopic surgeries, with no need of incision or prophylactic antibiotics [20]. In the study of Patel et al., he reported very similar graft success rates in patients undergoing endoscopic and microscopic tympanoplasty. $\mathrm{He}$ also reported that the major disadvantage of endoscopic approach was the necessity to operate with a single hand. They also stated that any bleeding in the external canal made manipulations very difficult and underlined the necessity to achieve to total hemostasis in the external ear canal. The mean time of endoscopic and microscopic tympanoplasty operation was found to be $75 \mathrm{~min}$ and $90 \mathrm{~min}$ respectively [21].

Huang et al. reported that the mean operation times were $75.5 \mathrm{~min}$ and $50.4 \mathrm{~min}$ in patients undergoing microscopic and endoscopic tympanoplasty, respectively. He performed type 1 tympanoplasty in 50 patients by microscopic approach and another 50 patients by endoscopic approach, and the results were similar of hearing recovery and rate of perforation closure were found between the two patient groups. Moreover, endoscopic approach group showed shorter operation times, better views of surgical field, improved outcomes, and reduced tissue injury [22].

Gerard and Gersdorff used tutopatch graft which is a xenograft derived from bovine pericardium for repair of mid-sized tympanic membrane perforations through transcanal approach without endoscope with similar success results to our study but they have the limitations of using a xenograft in anatomically accessible patients and mid-sized perforations [23].

\section{Conclusions}

This study revealed the efficacy and safety of endoscopic transcanal tympanoplasty using Tutoplast temporalis facia graft in comparison with the traditional postauricular microscopic surgery. Tutoplast graft is suitable for 
repair of tympanic membrane perforation of different size and shape. Endoscopic transcanal approach carries many advantages; good exposure of tympanic membrane; and magnified clear visualization of middle ear details.

Use of Tutoplast graft for endoscopic closure of tympanic membrane perforation is successful short-time procedure avoiding the postauricular or endaural incisions.

Few disadvantages have been reported for endoscopic tympanoplasty. Single-handed technique with risk of direct trauma and thermal injury, however, we believed that these disadvantages can be overcome by a welltrained surgeon using the endoscope with average light intensity and dimming operating room light.

\section{Abbreviations}

ORL: Oto-rhino-laryngology; PTA: Pure tone audiometry; ABG: Air bone gap; CT: Computed tomography; P: Posterior meatal wall; C: Chorda tympani; I: Incus; R: Round window niche; M: Malleus; F: Tympanomeatal flap;

T: Tutoplast; IS: Incudo-stapedial joint; G: Gelfoam

\section{Acknowledgements}

Not applicable.

\section{Authors' contributions}

A.A. analyzed and interpreted the patient data regarding the audiological results. T. M. was a major contributor in writing the manuscript. Both authors contributed in surgical procedures, read, and approved the final manuscript.

\section{Funding}

None

\section{Availability of data and materials}

The datasets used and/or analyzed during the current study are available from the corresponding author on reasonable request.

\section{Declarations}

\section{Ethics approval and consent to participate}

This study was conducted on 50 patients after getting approval from the ethical committee of medical research at NMC Royal Hospital, Abu Dhabi, UAE.

No reference number available.

A written consent from all patients to be enrolled in this study was obtained.

\section{Consent for publication}

Not applicable.

\section{Competing interests}

The authors declare that they have no competing interests.

Received: 30 April 2021 Accepted: 18 July 2021

Published online: 23 August 2021

\section{References}

1. Rourke T, Snelling JD, Aldren C (2010) Cartilage graft butterfly myringoplasty: how we do it. Clin Otolaryngol 35:135-138

2. Tarasov DI (1988) Disease of the middle ear. M Medicine 66:1076-1095

3. Westerberg J, Harder H, Magnuson B, Westerberg L, Hyden H (2011) Tenyear myringoplasty series: does the cause of the perforation affect the success rate. J Laryngol Otol 125:126-132

4. Albera R, Ferrero V, Lacilla M, Canale A (2006) Tympanic reperforation in myringoplasty: evaluation of prognostic factors. Ann Otol Rhinol Laryngol 115:875-879
5. Sergi B, Galli J, De Corso E, Parrilla C, Paludetti G (2011) Overlay versus underlay myringoplasty: report of outcomes considering closure of perforation and hearing function. Acta Otorhinolaryngol Ital 31:366-371

6. Zollner $F(1955)$ The principles of plastic surgery of the sound-conducting apparatus. J Laryngol Otol 69(10):637-652

7. Jyothi C, Shrikrishna H, Kulkarni NH, Kumar A (2017) Endoscopic myringoplasty versus microscopic myringoplasty in tubotympanic CSOM. Indian J Otolaryngol Head Neck Surg 69(3):357-362

8. Ayache S, Tramier B, Strunski V (2008) Otoendoscopy in cholesteatoma surgery of the middle ear: what benefits can be expected? Otol Neu- rotol 29(8):1085-1090

9. Harugop AS, Mudhol RS, Godhi RA (2008) A comparative study of endoscope assisted myringoplasty and microscope assisted myringoplasty. Indian J Otolaryngol Head Neck Surg 60:290-302

10. Patel J, et al (2015) Endoscopic tympanoplasty vs microscopic tympanoplasty in tubotympanic csom: a comparative study of 44 cases. Int J Res Med Sci 3(8):1953-1957

11. El-Guindy A (1992) Endoscopic transcanal myringoplasty. J Laryngol Otol 106:493-495

12. Mohindra S, Panda NK (2010) Ear surgery without microscope; is it possible. Indian J Otolaryngol Head Neck Surg 62:138-141

13. Sakallıoglum AAO, Karlidag T (2017) Review of endoscopic tympanoplasty. J Otol 12:62-67

14. Bottrill I, Perrault DF Jr, Poe D (1996) In vitro and in vivo determination of the thermal effect of middle ear endoscopy. Laryngoscope. 106(2 Pt 1):213216

15. Kozin ED, Lehmann A, Carter M, Hight E, Cohen M, Nakajima HH et al (2014) Thermal effects of endoscopy in a human temporal bone model: implications for endoscopic ear surgery. Laryngoscope. 124(8):E332-E339

16. Choi N, Noh Y, Park W, Lee JJ, Yook S, Choi JE, Chung W-H, Cho Y-S, Hong SH, Moon J (2017) Comparison of endoscopic tympanoplasty to microscopic tympanoplasty. Clin Exp Otorhinolaryngol 10(1):44-49

17. Kumar S, Kumar A (2017) Endoscopic type I tympanoplasty in medium sized tympanic membrane perforation: our experience in tertiary care center IOSR. J Dent Med Sci 16(2) Ver. III:58-60

18. Dornhoffer UL (1997) Hearing results with cartilage tympanoplasty. Laryngoscope. 107(8):1094-1099

19. Mokbel KM, Moneir W, Elsisi H, Alsobky A (2015) Endoscopic transcanal cartilage myringoplasty for repair of subtotal tympanic membrane perforation: a method to avoid postauricular incision. J Otolaryngol Rhinol 1:010

20. Awad OG, Hamid KA (2015) Endoscopic type 1 tympanoplasty in pediatric patients using tragal cartilage. JAMA Otolaryngol Head Neck Surg 141(6): 532e538

21. Patel J, Aiyer RG, Gajjar Y, Gupta R, Raval J, Suthar PP (2015) Endoscopic tympanoplasty vs microscopic tympanoplasty in tubotympanic CSOM: a comparative study of 44 cases. Int J Res Med Sci 3(8):1953-1957

22. Huang TY, Ho KY, Wang LF, Chien CY, Wang M (2016) A comparative study of endoscopic and microscopic approach type 1 tympanoplasty for simple chronic otitis media. J Int Adv Otol 12(1)

23. Gerard JM, Gersdorff (2006) The Tutopatch graft for transcanal myringoplasty. B-ENT 2(4):177-179

\section{Publisher's Note}

Springer Nature remains neutral with regard to jurisdictional claims in published maps and institutional affiliations. 